\title{
Circulating interleukin 6 and interleukin 10 in community acquired pneumonia
}

\author{
P Glynn, R Coakley, I Kilgallen, N Murphy, S O’Neill
}

\begin{abstract}
Background-Inflammatory cytokine concentrations correlate with severity of sepsis. We hypothesised that patients with community acquired pneumonia (CAP) associated with systemic inflammatory response syndrome (SIRS) would have greater interleukin 6 (IL-6) production due to activation of the inflammatory cytokine cascade, matched by a significant anti-inflammatory cytokine response. Interleukin 10 (IL-10) was evaluated as a potential surrogate marker of severity of sepsis in CAP and age related impairment of the cytokine response was studied in elderly patients with CAP.

Methods-Circulating immunoreactive IL-6 and IL-10 levels were measured in 38 patients with CAP subdivided into a group fulfilling the criteria for SIRS $(n=28)$ and a non-SIRS group $(n=10)$ in a variety of age groups and correlated with APACHE II scores.

Results-80\% had circulating IL-6 levels (median $46.7 \mathrm{pg} / \mathrm{ml}$, range $4.6-27000$ ) and $60 \%$ had circulating IL-10 levels (median $15.5 \mathrm{pg} / \mathrm{ml}$, range $2.5-765)$. Concentrations of both were significantly increased in patients with SIRS compared with nonSIRS patients. Those with activation of the inflammatory cytokine cascade (IL-6 positive) produced more IL-10 than IL-6 negative patients. Older patients had a similar cytokine response. Both cytokines correlated positively with APACHE II scores.
\end{abstract}

Conclusions-This is the first demonstration of circulating IL-10 in CAP. A greater counter-inflammatory response in patients with SIRS and in IL-6 positive patients suggests a potential immunomodulatory role for IL-10 in controlling the inflammatory cytokine response in CAP. IL-10 concentrations correlate with severity of illness in CAP and may be of prognostic importance. There is no age related impairment in the cytokine response.

(Thorax 1999;54:51-55)

Keywords: interleukin 6; interleukin 10; community acquired pneumonia

Community acquired pneumonia (CAP) still ranks amongst the five major causes of death worldwide despite the availability of potent antibiotic therapy. The mortality rate in hospitalised patients ranges from $10 \%$ to $25 \%$ and is higher still in patients requiring admission to ITU. ${ }^{1}$

Release of pro-inflammatory cytokines, particularly IL-1, IL-6, IL-8, and TNF- $\alpha$, from activated monocytes and pulmonary macrophages is an important component of the host immune response and the role of these molecules in the pathogenesis of sepsis has been well studied. ${ }^{23}$ In many studies of sepsis, overproduction of pro-inflammatory cytokines has been demonstrated and concentrations shown to correlate with severity and outcome of sepsis. ${ }^{4}$ In particular, excess production of IL-6 - which is believed to be a good marker of an exaggerated pro-inflammatory responsecorrelates with APACHE II scores ${ }^{6}$ and increased mortality.

The host pro-inflammatory cytokine response in pneumonia is, to a large extent, compartmentalised to the affected lung, ${ }^{8}$ but circulating inflammatory cytokines have also been detected in peripheral blood and Puren et al found a positive correlation between systemic IL-6 concentrations and APACHE II scores. ${ }^{9}$ They failed to show an association between cytokine levels and mortality.

In sepsis, inflammatory stimuli also activate production of specific cytokine neutralising molecules such as soluble TNF receptors, and counter-inflammatory cytokines such as IL-10 that can downregulate the host inflammatory response, and may have a key role to play in controlling the pro-inflammatory cytokine response. Interleukin-10 production in vitro is stimulated by endotoxin and inhibits monocyte release of a variety of pro-inflammatory cytokines. ${ }^{10} \mathrm{~A}$ potent anti-inflammatory cytokine, it is detectable in the plasma of patients with sepsis, ${ }^{11}$ with higher levels in those with septic shock. ${ }^{12}$ Other studies have also confirmed this ${ }^{13}$ and have shown that nonsurvivors of sepsis had persistently raised levels of IL-10, while in survivors the IL-10 levels decreased over time. ${ }^{14}$ Deficiency of local intrapulmonary IL-10 in early ARDS appears to confer a poor prognosis, ${ }^{15}$ emphasising its immunomodulatory role in inflammation. Indeed, the anti-inflammatory response should be a good predictor of outcome in sepsis because of its critical role in controlling the cytokine cascade.

Soluble TNF receptor concentrations are increased in community acquired bacterial pneumonia, but relatively less so than in patients with septic shock. ${ }^{16}$ Interleukin 10 , the most potent anti-inflammatory cytokine, has not yet been studied in CAP. The primary aim of this study was to determine if there is production and systemic circulation of IL-10 in CAP, and to evaluate this anti-inflammatory cytokine in relation to the pro-inflammatory cytokine response, for which systemic IL-6 is a marker. 
Community acquired pneumonia has a spectrum of presentations with a significant proportion of patients, particularly the elderly, lacking the classical features of infection, ${ }^{17}$ and a minority developing septic shock and multiorgan failure. We subdivided our patients according to whether or not they fulfilled standard criteria for the systemic inflammatory response syndrome (SIRS). ${ }^{18}$ We postulated that SIRS secondary to pneumonia would reflect an underlying pro-inflammatory cytokine response, and that the anti-inflammatory response in terms of IL-10 production would be greater in patients with SIRS secondary to pneumonia than in those who lacked the classical features of sepsis. The prognostic significance of IL-10 concentrations in CAP was also evaluated.

Finally, since features of infection such as fever, tachycardia, and neutrophilia have been shown to be absent in over a third of elderly patients with $\mathrm{CAP},{ }^{17}$ we postulated that older patients with pneumonia may have a functional immunological impairment in terms of inflammatory/anti-inflammatory cytokine response to infection manifesting as a failure to exhibit classic features of sepsis. Support for the concept of host immunosenescence impairing pulmonary inflammatory responses in the elderly comes from evidence of suboptimal accessory cell cytokine production in aged mice. ${ }^{19}$ In particular, there is a relative reduction in macrophage production of IL-1 essential to drive $\mathrm{T}$ cell proliferation. It has also been shown that healthy elderly individuals have impaired neutrophil effector functions such as chemotaxis and phagocytosis. ${ }^{20}$

\section{Methods}

The study took place in the Department of Respiratory Medicine, Beaumont Hospital, Dublin. We studied 38 patients with CAP consecutively admitted through the Accident and Emergency Department. A diagnosis of CAP was made when the patient had all of the following: a new radiological infiltrate, a dry or productive cough of recent onset, a pyrexia and signs of lung consolidation (coarse crackles and/or bronchial breath sounds) corresponding to the radiographic findings for which no other cause could be found. We excluded patients who were immunocompromised. Two groups of patients were studied: those who had features on initial assessment of systemic inflammatory response syndrome (SIRS; $\mathrm{n}=$ $28)$ and those who did not $(n=10)$. The inclusion criteria for the SIRS group were three or more of the following: temperature $>38^{\circ} \mathrm{C}$ or $<36^{\circ} \mathrm{C}$, heart rate $>90$ beats/min, respiratory rate $>20$ breaths $/ \mathrm{min}$ or $\mathrm{PaCO}_{2}<4.3 \mathrm{kPa}$, and white blood cell count of $>12000$ cells $/ \mathrm{mm}^{3}$, $<4000$ cells $/ \mathrm{mm}^{3}$, or $>10 \%$ immature (band) forms. Patients were given standard empirical antibiotic therapy according to the American Thoracic Society guidelines ${ }^{21}$ and appropriate supportive treatment. The decision to manage one patient with SIRS in the ITU was based on the requirement for mechanical ventilation and this patient subsequently died. Severity of illness was assessed using the acute physiology and chronic health evaluation (APACHE) II index. We also studied a group of 25 age matched healthy controls. Patient data collected included demographic data, chest radiographic findings, routine laboratory data, arterial blood gas tensions, and blood cultures. For measurement of cytokine concentrations clotted blood samples were centrifuged immediately at $2500 \mathrm{rpm}$ for 10 minutes and serum stored at $-80^{\circ} \mathrm{C}$. Circulating immunoreactive IL-6 and IL-10 levels were measured using commercially available quantitative enzymelinked immunosorbent assays (ELISA, R\&D Systems Europe, Abingdon, UK). The assays did not measure biological activity of the cytokines. Standard sensitivity assays were used and the manufacturers reported the sensitivity thresholds in serum as $0.7 \mathrm{pg} / \mathrm{ml}$ and $1.5 \mathrm{pg} / \mathrm{ml}$ for IL-6 and IL-10, respectively. All measurements were made by a single trained individual to avoid interobserver variation. All samples were assayed in duplicate.

\section{STATISTICAL ANALYSIS}

Calculations were carried out using the statistical software package GraphPad Prism Version 2.0 (GraphPad Software Inc, San Diego, California) and non-parametric statistical tests were used for comparison of cytokine concentrations, in particular the Mann-Whitney U test and the $\chi^{2}$ test. Spearman's correlation coefficient was used to determine correlation between APACHE II scores and cytokine concentrations, expressing data on a log scale. Unpaired $t$ tests were used for analysis of data found to be normally distributed including data such as temperature, pulse rate, and white cell count. The results are expressed as mean (SE).

For comparison of cytokine concentrations in elderly and younger patients with pneumonia, only those over 70 years $(n=18)$ and under 60 years $(n=11)$ were included in the analysis to allow a clear demarcation between older and younger patients.

\section{Results}

A total of 38 patients were studied. Of these, 28 fulfilled two or more necessary criteria for SIRS on admission and the remaining $10 \mathrm{did}$ not. No patients met the criteria for severe sepsis or septic shock. Patients with SIRS had significantly higher pulse rates $(p<0.0005)$ and respiratory rates $(p<0.05)$ than patients without SIRS, but non-significantly higher temperatures and white cell counts (table 1). Patient age was similar in the two groups. The

Table 1 Mean (SE) clinical and laboratory data in patients with community acquired pneumonia (CAP) with and without the systemic inflammatory response syndrome (SIRS)

\begin{tabular}{lll}
\hline & SIRS & Non-SIRS \\
\hline Age & 66 & 62 \\
Respiratory rate & $26.2(1.4)^{\star}$ & $20.3(1)$ \\
Pulse rate & $104(3)^{\star \star}$ & $82.7(2)$ \\
White cell count & $18.4(2)$ & $14(2)$ \\
Temperature & $38(0.2)$ & $37.4(0.2)$ \\
APACHE II & $13(2)$ & $9(2)$
\end{tabular}

${ }^{\star} \mathrm{p}<0.05 ;{ }^{\star \star} \mathrm{p}<0.0005$. 

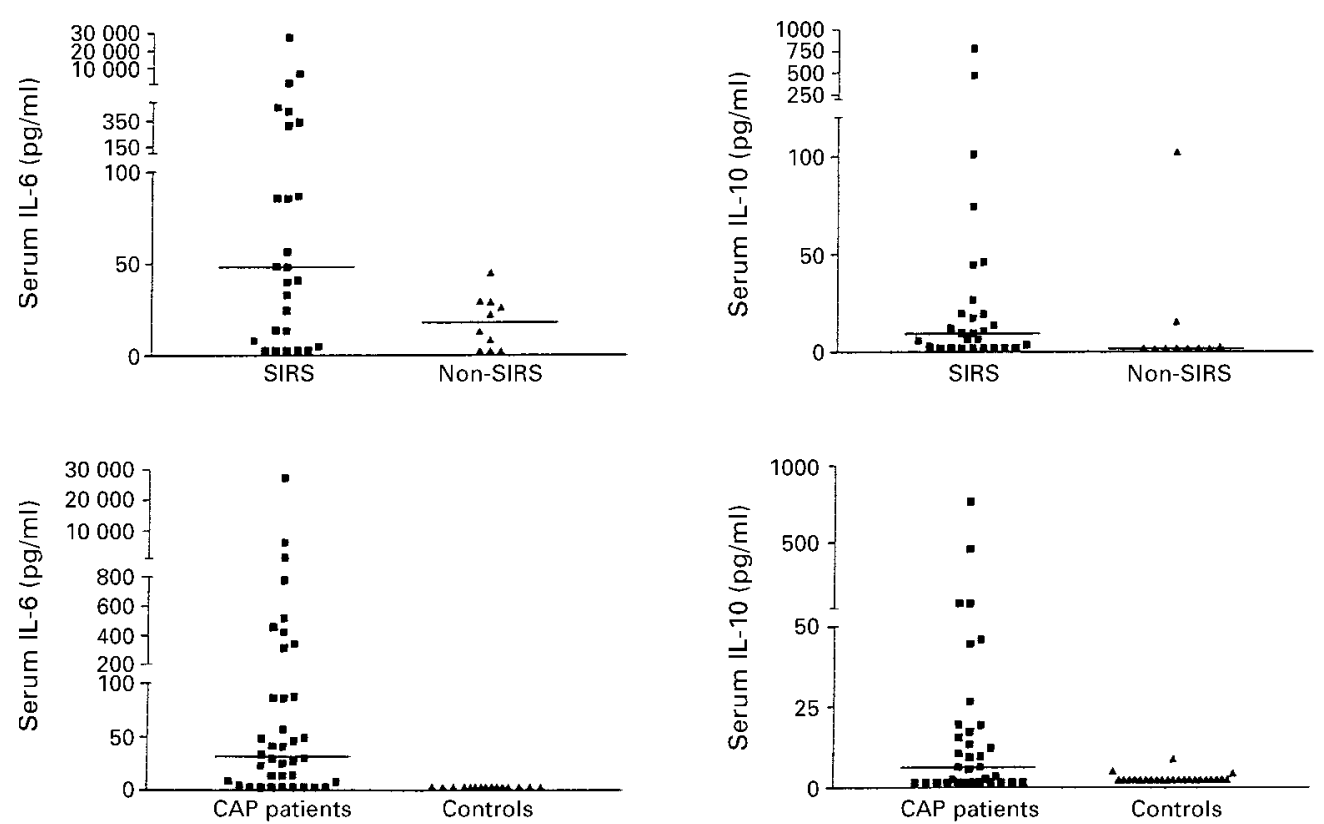

Figure 1 IL-6 and IL-10 concentrations in patients with community acquired pneumonia (CAP) and controls. Median bars are shown.

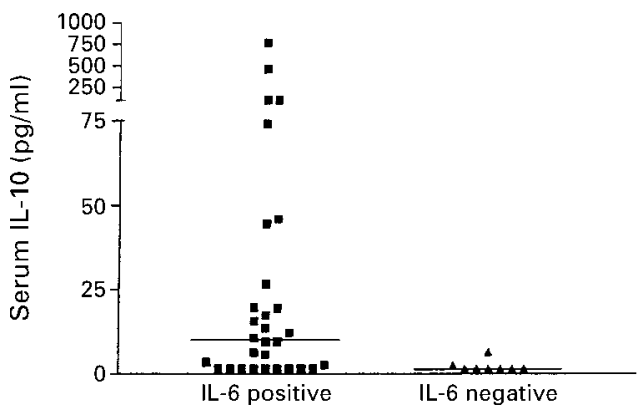

Figure 2 IL-10 concentrations in IL-6 positive and IL-6 negative patients with community acquired pneumonia $(C A P)$. Median bars are shown.

mean APACHE II score was 12 (range 2-34) and APACHE II scores were similar in both subgroups. Respiratory complications included parapneumonic effusion (3), empyema (2), and respiratory failure (13), all in the SIRS group. One patient had underlying bronchogenic carcinoma. There were four deaths related to the primary diagnosis of pneumonia, all in the SIRS group. Three patients died after the study period (carcinoma of tongue, bronchogenic carcinoma, and an isolated second episode of pneumonia).

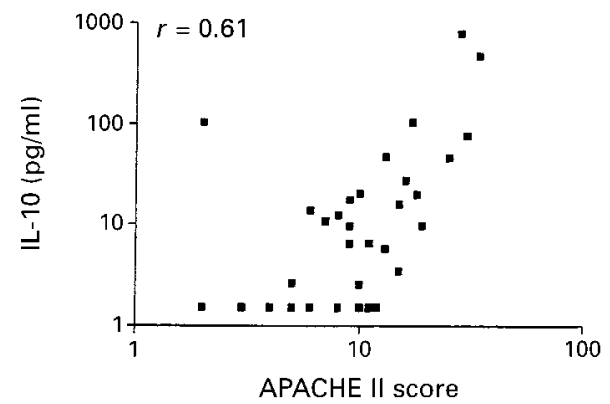

CYTOKINE DATA

Our sensitivity threshold for the IL-6 assay was $2.3 \mathrm{pg} / \mathrm{ml}$. This quantity was detected in a healthy control and we did not detect the minute quantities reported by the manufacturers $(0.7$ $\mathrm{pg} / \mathrm{ml}$ ) in any individual. However, 30 of the 38 patients $(80 \%)$ had circulating IL-6 levels detectable on admission (median $46.7 \mathrm{pg} / \mathrm{ml}$, range 4.6-27 000; fig 1) compared with two of 17 healthy controls $(\mathrm{p}<0.005)$. The remaining eight patients had extremely low circulating IL-6 concentrations $(<2.5 \mathrm{pg} / \mathrm{ml})$ or none at all. Our sensitivity threshold for the IL-10 assay was $1.5 \mathrm{pg} / \mathrm{ml}$, identical to the figure reported by the manufacturers. Twenty three of the 38 patients $(60 \%)$ had detectable IL-10 levels (median $15.5 \mathrm{pg} / \mathrm{ml}$, range $2.5-765 \mathrm{pg} / \mathrm{ml}$; fig 1) compared with four of 25 healthy controls $(p<0.005)$. Looking more closely at the patients with pneumonia, we found that those with SIRS had significantly higher concentrations of both IL-6 and IL-10 than the non-SIRS group (fig 1). Only two of the latter group had detectable IL-10 concentrations.

We found significant differences in IL-10 concentrations between patients with increased IL-6 levels on admission $(n=30)$ and those with undetectable IL-6 $(n=8)$. Concentrations

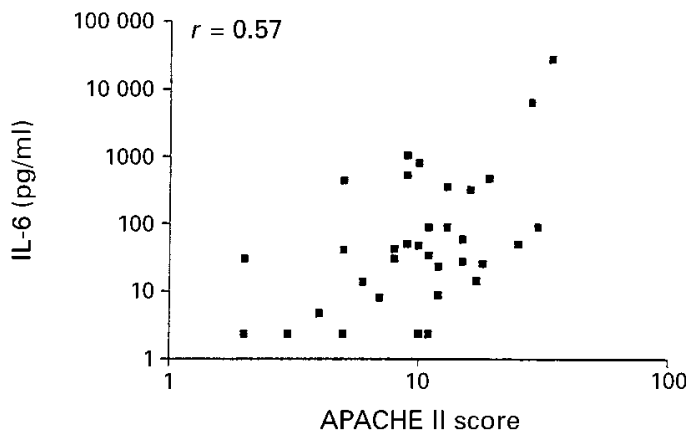

Figure 3 Correlation between APACHE II scores and IL-10 and IL-6 concentrations. 
Table 2 Mean (SD) IL-6 and IL-10 concentrations in patients aged over 70 years and under 60 years

\begin{tabular}{lll}
\hline & $I L-6(p g / m l)$ & $I L-10(p g / m l)$ \\
\hline Patients $>70$ years & $1995(6404)$ & $80(201)$ \\
Patients $<60$ years & $52.6(117)$ & $11.8(29)^{\star}$ \\
\hline${ }^{\star} \mathrm{p}<0.05$. & &
\end{tabular}

of IL-10 were significantly higher in the former group (fig 2). In fact, there was virtually no IL-10 production in the "IL-6 negative" patients (fig 2). There was a positive correlation between levels of IL- 6 and IL-10 in our study patients $(r=0.53, \mathrm{p}<0.001)$.

Cytokine levels were not influenced by the duration of infection prior to performing the measurements (as judged by duration of symptoms).

There was strong positive correlation between concentrations of both IL- 6 and IL-10 and patient APACHE II scores $(r=0.57$ and $r$ $=0.61$, respectively; fig 3 ). Smokers and non-smokers with pneumonia had similar cytokine levels.

Concentrations of IL-10 were similar in patients over $70(n=18)$ and under $60(n=11)$ years of age, and IL-6 levels (table 2) were actually higher in the elderly group $(\mathrm{p}<0.05)$.

\section{Discussion}

The presence of circulating pro-inflammatory cytokines in patients with pneumonia has been well described, but this is the first study to demonstrate systemic circulation of the counter-inflammatory cytokine IL-10 in a majority of patients with CAP.

The positive correlation between IL- 6 and IL-10 concentrations may reflect a shared underlying stimulus to their production, such as LPS or TNF- $\alpha$, circulating in response to the primary infection, or may indicate that proand anti-inflammatory cytokines are coregulated in some other way. Other authors have also found a correlation between proinflammatory and anti-inflammatory cytokines in sepsis, ${ }^{12}$ and have reported the correlation between TNF and IL-10 to be even stronger than between IL- 6 and IL-10, supporting our hypothesis that the production of antiinflammatory cytokines during sepsis is proportional to the inflammatory response.

We have shown that patients with SIRS secondary to pneumonia, even in the absence of severe sepsis or shock, have a greater underlying pro-inflammatory cytokine response than those who lack the features of sepsis. We have not elicited the source of the circulating cytokines in this study, but other authors have shown that the inflammatory cytokine response in pneumonia is largely compartmentalised to the affected lung. ${ }^{8}$ Dehoux et al showed that serum levels of IL- 6 correlated positively with IL-6 concentrations in bronchoalveolar lavage (BAL) fluid from the involved side. "Spillover" of cytokines from the pulmonary vasculature into circulating blood after local production in the lung is the most likely scenario, but extrapulmonary production by circulating monocytes primed by exposure to circulating inflammatory stimuli is also possible. Unfortu- nately we do not have data available on cytokine concentrations in the BAL fluid of these patients so we cannot be more conclusive about the source of circulating IL-10. If local pulmonary production could be assumed, then a stronger correlation between BAL fluid cytokine concentrations and the clinical features of sepsis would be anticipated. However, in this study which was confined to circulating cytokine measurements, serum IL-6 concentrations do seem to mirror the clinical features of sepsis in pneumonia as higher concentrations of IL- 6 and also IL-10 have been detected in patients with features of SIRS. These septic patients may produce larger quantities of the anti-inflammatory cytokine as a protective host mechanism to balance the systemic inflammatory response. In support of this our data show that patients with circulating IL-6 (a marker of activation of the inflammatory cytokine cascade) also have the highest concentrations of IL-10, and patients with no circulating IL-6 on admission have virtually no detectable circulating IL-10. This concurs with similar findings in paediatric sepsis in which it has been shown that the highest IL-10 concentrations are found in patients with high circulating IL-6 and nitrite/nitrate concentrations, ${ }^{22}$ and that these patients also have increased organ failure. Thus, IL-10 may only circulate systemically in pneumonia in significant amounts when there is a large systemic inflammatory response, possibly as a mechanism to control the inflammatory cytokine response. If we have missed very small circulating quantities of IL-6 in some individuals due to suboptimal sensitivity of the assay, then this effect can only have been negligible. Accepting that we lack data on the temporal pattern of cytokine secretion, the overall impression from this study is of an appropriate balance between pro- and anti-inflammatory cytokine production in pneumonia.

There are a number of other specific anti-inflammatory effects of IL-10 that may be relevant in CAP. IL-10 inhibits neutrophil production of pro-inflammatory cytokines ${ }^{23}$ including IL-8, a major neutrophil chemotactic factor. Neutrophil accumulation in the lung in pneumonia is amplified by local production of IL-8, which has been shown to be compartmentalised to the affected lung leading to a local elastase/anti-elastase imbalance. ${ }^{24}$ Since parenchymal damage may be mediated in part by this neutrophil derived elastase burden, the factors regulating local IL-8 production in the lung are of obvious importance. In animal models of acute lung injury IL-10 shortens the period of pulmonary neutrophilia induced by LPS challenge and a role has been shown for IL-10 in enhancing resolution of pulmonary inflammation by promoting apoptosis of neutrophils. ${ }^{25}$ The importance of IL-10 in pulmonary inflammation is also supported by the findings of Donnelly et al who showed that a local intrapulmonary deficiency of IL-10 in ARDS appears to confer a poor prognosis. ${ }^{15}$

While it is well established that proinflammatory cytokines may be good predictors of both morbidity and mortality in sepsis, systemic IL-6 concentrations have not been 
found useful in predicting the outcome in CAP. We have confirmed the findings of previous authors ${ }^{9}$ demonstrating a positive correlation between IL-6 levels and APACHE II scores, but this is the first study to show a similar association between IL-10 concentrations and APACHE II scores. It was not possible to differentiate between our two subgroups with respect to morbidity as APACHE II scores were not significantly different, although four patients with SIRS had respiratory complications such as empyema and parapneumonic effusion which did not occur in the non-SIRS group. This probably reflects a multitude of factors such as type of bacterial infection and choice of antibiotics rather than severity of infection per se. The absolute number of fatalities secondary to pneumonia in this study was relatively low, but these patients had significantly higher concentrations of both cytokines. This, in conjunction with the above data relating the cytokine response to patient morbidity, supports a possible immunomodulatory role for pro-inflammatory and anti-inflammatory cytokines in the pathogenesis of CAP. It certainly emphasises the need for further evaluation of the anti-inflammatory cytokine response in severe and fatal pneumonia.

Patients with SIRS in CAP were not, as we hypothesised, significantly younger than the non-SIRS group. While elderly patients were less likely to develop a fever of more than $38^{\circ} \mathrm{C}$ $(44 \%)$, a leucocytosis was found in two thirds of older patients. There was therefore a wide spectrum of presentation among the older patients with many of them mounting an appropriate clinical response to their infection. Overall, patients aged over 70 years had higher levels of IL- 6 than those under 60 and similar concentrations of IL-10 (table 2). We could find no evidence of age related immunosenescence in terms of systemic cytokine response in this group of patients with CAP.

In summary, we have demonstrated systemic IL-6 and IL-10 levels in most of our patients with CAP and found that those with a systemic inflammatory response to pulmonary infection (SIRS) have greater production of both proinflammatory and anti-inflammatory cytokines. Patients with circulating IL-6 produce greater amounts of IL-10 than those in whom there is no detectable IL-6, which supports our hypothesis that in pneumonia the counterinflammatory response is greatest when there is activation of the pro-inflammatory cytokine cascade, perhaps as a protective mechanism. Interleukin-10 may have other important immunomodulatory effects relevant to enhancement or resolution of pulmonary neutrophilic inflammation.

This is the first study to find a relationship between IL-10 production and severity of illness in CAP, giving supportive evidence for a possible immunomodulatory role and suggesting its potential use as a prognostic marker in severe pneumonia.

Addressing the issue of host immunosenescence as a feature of and possibly contributing to the severity of pneumonia in elderly patients, we have found these patients to be largely capable of mounting a systemic inflammatory response to infection, and this is reflected in their ability to generate appropriate quantities of both pro-inflammatory and antiinflammatory cytokines.

1 Torres A, Serra-Battles J, Ferrer A, et al. Severe communityacquired pneumonia: epidemiology and prognostic factors. Am Rev Respir Dis 1991;144:312-8.

2 Calandra T, Gerain J, Heumann D, et al and the Swiss-Dutch J5 Immunoglobulin Study Group. High circulating levels of interleukin-6 in patients with septic shock: evolution during sepsis, prognostic value and interplay with other cytokines. Am F Med 1991;91:23-9.

3 Cannon JG, Tompkins RG, Gelfland JA, et al. Circulating interleukin-1 and tumour necrosis factor in septic shock and experimental endotoxin fever. F Infect Dis 1990;160: $79-84$.

4 Endo S, Inada $\mathrm{K}$, Inoue $\mathrm{Y}$, et al. Two types of septic shock classified by the plasma levels of cytokines and endotoxin. Circulatory Shock 1992;38L:264-74.

5 Martin C, Sauzx P, Mege JL, et al. Prognostic value of serum cytokines in septic shock. Intensive Care Med 1994;20:2727 .

6 Knaus WA, Draper EA, Douglas PW, et al. APACHE II: a severity of disease classification system. Crit Care Med 1985;13:818-29.

7 Damas P, Ledoux D, Nys M, et al. Cytokine serum levels during severe sepsis in humans: IL-6 as a marker of severity. Ann Surg 1992;215:356-62.

8 Dehoux M, Boutten A, Ostinelli J, et al. Compartmentalised cytokine production within the human lung in unilateral pneumonia. Am f Respir Crit Care Med 1994;150:710-6.

9 Puren J, Feldman C, Savage N, et al. Patterns of cytokine expression in community-acquired pneumonia. Chest 1995;107:1342-9.

10 De Waal Malefyt, Abrams RJ, Bennett B, et al. Interleukin 10 (IL-10) inhibits cytokine synthesis by human 10 (IL-10) inhibits cytokine synthesis by human monocytes: an autoregulatory role of IL-10

11 Andrew F, Riordan I, Marzouk O, et al. Proinflammatory and anti-inflammatory cytokines in meningococcal disease. Arch Dis Child 1996;75:453-4.

2 Marchant A, Deviere J, Byl B, et al. Interleukin-10 production during septicaemia. Lancet 1994;343:707-8.

13 Jiminez J, Martin MC, Sauri R, et al. Interkeukin-10 and the monocyte/macrophage-induced inflammatory response in septic shock. F Infect Dis 1995;171:472-5.

14 van der Poll T, de Waal Malefyt R, Coyle SM, et al. Anti-inflammatory cytokine response during clinical sepsis and experimental endotoxemia: sequential measurements of plasma soluble interleukin (IL)-1 receptor type II, IL-10 and IL-13. F Infect Dis 1997; 175:118-22.

15 Donnelly S, Strieter R, Reid P, et al. The association between mortality rates and decreased concentrations of interleukin-10 and interleukin-1 receptor antagonist in the lung fields of patients with the adult respiratory distress lung fields of patients with the adult respirat
syndrome. Ann Intern Med 1996;125:191-6.

16 de Werra I, Jaccard C, Corradin SB, et al. Cytokines, nitrite/ nitrate, soluble tumour necrosis factor receptors, and procalcitonin concentrations: comparisons in patients with septic shock, cardiogenic shock and bacterial pneumonia. Crit Care Med 1997;25:607-13.

17 Venkatesan P, Gladman J, Macfarlane JT, et al. A hospital study of community acquired pneumonia in the elderly. Thorax 1990;45:254-8.

18 American College of Chest Physicians/Society of Critical Care Medicine Consensus Conference. Definitions for sepsis and organ failure and guidelines for the use of innovative therapies in sepsis. Crit Care Med 1992;20:864-73.

19 Inamizu T, Chang M, Makinodan T. Influence of age on the production and regulation of interleukin-1 in mice. Immunology 1985;55:447.

20 Polignano A, Torterella C, Venezia A, et al. Age-associated changes of neutrophil responsiveness in a human elderly population. Cytobios 1994;80:144-53

21 Niederman MS, Bass JB, Campbell GD, et al. Guidelines for the initial management of adults with community-acquired pneumonia: diagnosis, assessment of severity, and initial antimicrobial therapy. Am Rev Respir Dis 1993;148:141826.

22 Doughty L, Kaplan S, Carcillo J. Inflammatory cytokine and nitric oxide responses in pediatric sepsis and organ failure. Crit Care Med 1996;24:1137-43.

23 Cassatella MA, Meda L, Bonora S, et al. IL-10 inhibits the release of proinflammatory cytokines from human polymorphonuclear leukocytes. Evidence for an autocrine role of tumour necrosis factor and IL- $1 \beta$ in mediating the production of IL-8 triggered by lipopolysaccharide. $\mathcal{F}$ Exp Med duction of IL-8 trigg

24 Boutten A, Dehoux M, Seta N, et al. Compartmentalized IL-8 and elastase release within the human lung in unilateral pneumonia. Am J Respir Crit Care Med 1996;153:33642 .

25 Cox G. IL-10 enhances resolution of pulmonary inflammation in vivo by promoting apoptosis of neutrophils. $A m \mathcal{F}$ Physiol 1996;271:L566-71. 